\title{
Tuberculous Osteomyelitis of Frontal Bone with Orbital Involvement: A Rare Case Presentation
}

\author{
${ }^{1}$ Sohil Gala, ${ }^{2}$ Vicky Khattar, ${ }^{3}$ Bachi Hathiram
}

\begin{abstract}
Introduction: Skeletal tuberculosis accounts for 1 to $3 \%$ of total cases of tuberculosis, out of which only 0.2 to $1.3 \%$ involve the calvarial bones, thus making it extremely rare.
\end{abstract}

Materials and methods: We discuss a case of tuberculous osteomyelitis of the frontal bone with orbital involvement. The patient was effectively managed by antituberculous therapy with no need for any surgical intervention.

Conclusion: With the advent of antituberculous chemotherapy and advanced diagnostic modalities, tuberculosis can be actively tackled, but still continues to be a leading public health problem in developing countries. A high index of suspicion in atypical cases must be considered.

Keywords: Frontal sinus, Orbital, Osteomyelitis, Tuberculosis.

How to cite this article: Gala S, Khattar V, Hathiram B. Tuberculous Osteomyelitis of Frontal Bone with Orbital Involvement: A Rare Case Presentation. Int J Otorhinolaryngol Clin 2018;10(1):32-34.

\section{Source of support: Nil}

Conflict of interest: None

\section{INTRODUCTION}

Tuberculosis continues to be among the greatest health problems in developing countries and has an enormous social and economic impact. Skeletal tuberculosis accounts for about 1 to $3 \%$ of total cases of tuberculosis. Tuberculous involvement of the skull is a rare entity and calvarial involvement is seen in only 0.2 to $1.3 \%$ cases of skeletal tuberculosis. ${ }^{1-3}$ Calvarial tuberculosis may have atypical presentations and may pose a diagnostic dilemma. We report a rare case of tuberculous involvement of the frontal bone with a discharging sinus and ocular involvement.

\footnotetext{
${ }^{1}$ Specialty Medical Officer, ${ }^{2}$ Assistant Professor, ${ }^{3}$ Professor and Head

${ }^{1-3}$ Department of ENT and Head and Neck Surgery, Topiwala National Medical College \& B.Y.L. Nair Ch. Hospital, Mumbai Maharashtra, India

Corresponding Author: Vicky Khattar, Assistant Professor Department of ENT and Head and Neck Surgery, Topiwala National Medical College \& B.Y.L. Nair Ch. Hospital, Mumbai Maharashtra, India, e-mail: orlclinics@gmail.com
}

\section{CASE REPORT}

A 60-year-old Indian male presented to us with a painless discharging sinus over the medial aspect of the left supraorbital ridge since 3 months and ophthalmic complaints since 10 days. There was intermittent cheesy discharge from the sinus which was occasionally blood-stained. He complained of blurring of vision, redness of the left eye with discharge, and proptosis since 10 days (Fig. 1). On examination, there was the presence of a single painless sinus opening over the medial aspect of left supraorbital ridge which exuded cheesy material on gentle pressure over the surrounding region. The left eye showed proptosis, upper lid edema, conjunctival inflammation, chemosis, and exposure keratitis of the cornea, with minimal restriction of extraocular movements. The vision was $6 / 18$ and a fundus examination could not be performed. Rest of the ear, nose, throat, and systemic examination revealed no abnormality.

The patient had a slightly raised erythrocyte sedimentation rate of $34 \mathrm{~mm}$ at the end of first hour by Wintrobe's method. The other blood parameters were normal.

A computed tomography of the paranasal sinuses and the orbit was performed, which revealed an osteolytic, peripherally enhancing soft tissue in the left frontal sinus extending to the left ethmoids with focal defect of the left lamina papyracea. Also seen were thickening and enhancement of the coat of the left eyeball with enhancement of the extraocular muscles of the left orbit. There was a defect in the superomedial aspect of left frontal sinus communicating with the overlying skin and soft tissue (Fig. 2).

The discharge from the sinus tract was sent for microbiological examination, which showed the presence of acid-fast bacilli on Ziehl-Neelsen staining (Fig. 3A). Bacteriological microscopy and culture studies were negative. Scrapings from the sinus wall revealed caseating granuloma with epithelioid and Langhan's giant cells on histopathology (Fig. 3B).

A final diagnosis of tuberculous osteomyelitis of the left frontal bone with orbital involvement was made.

The patient was started on multidrug antituberculous regimen with intensive phase with four drugs for 2 months followed by three drugs in continuation phase for 7 months, as per the recommended guidelines. The eye signs and symptoms improved in the first month and the sinus healed in 12 weeks after initiation of therapy. Besides an optical keratoplasty, no other surgical intervention was warranted. 

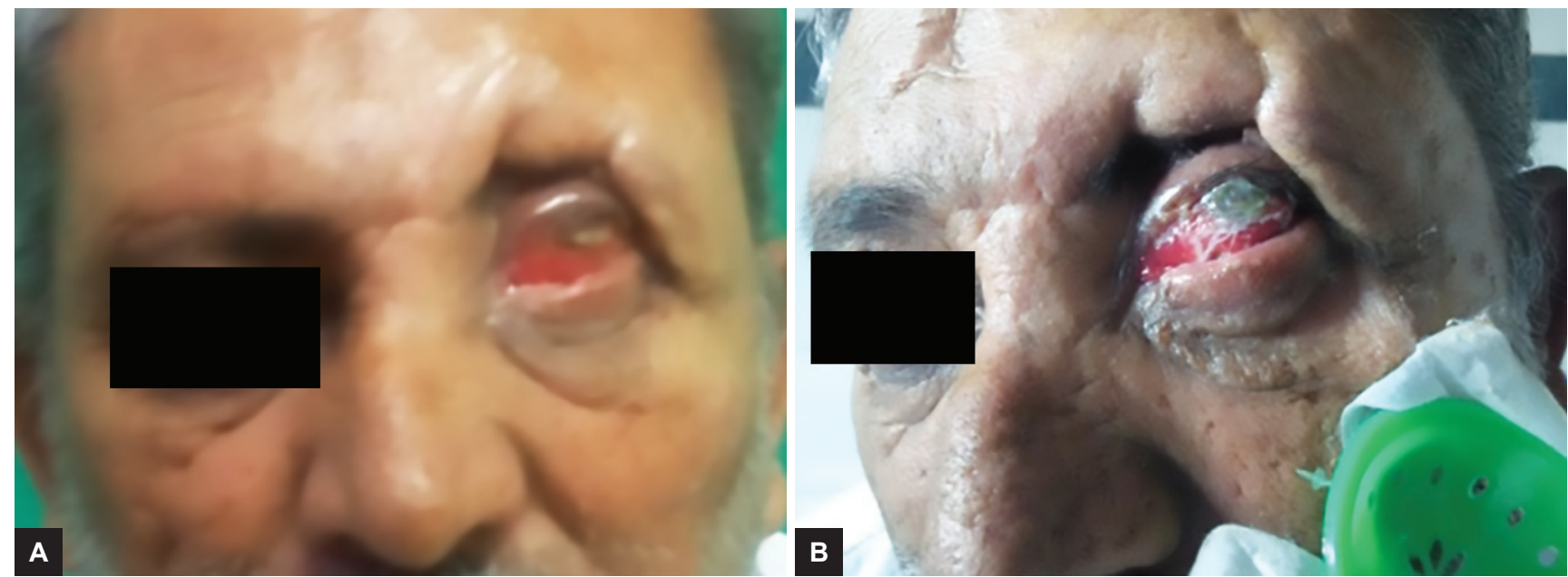

Figs $1 \mathrm{~A}$ and $\mathrm{B}$ : Clinical photograph showing a discharging sinus over the medial aspect of the left supraorbital ridge, with left eye proptosis, upper lid edema, conjunctival inflammation, chemosis, and exposure keratitis of the cornea
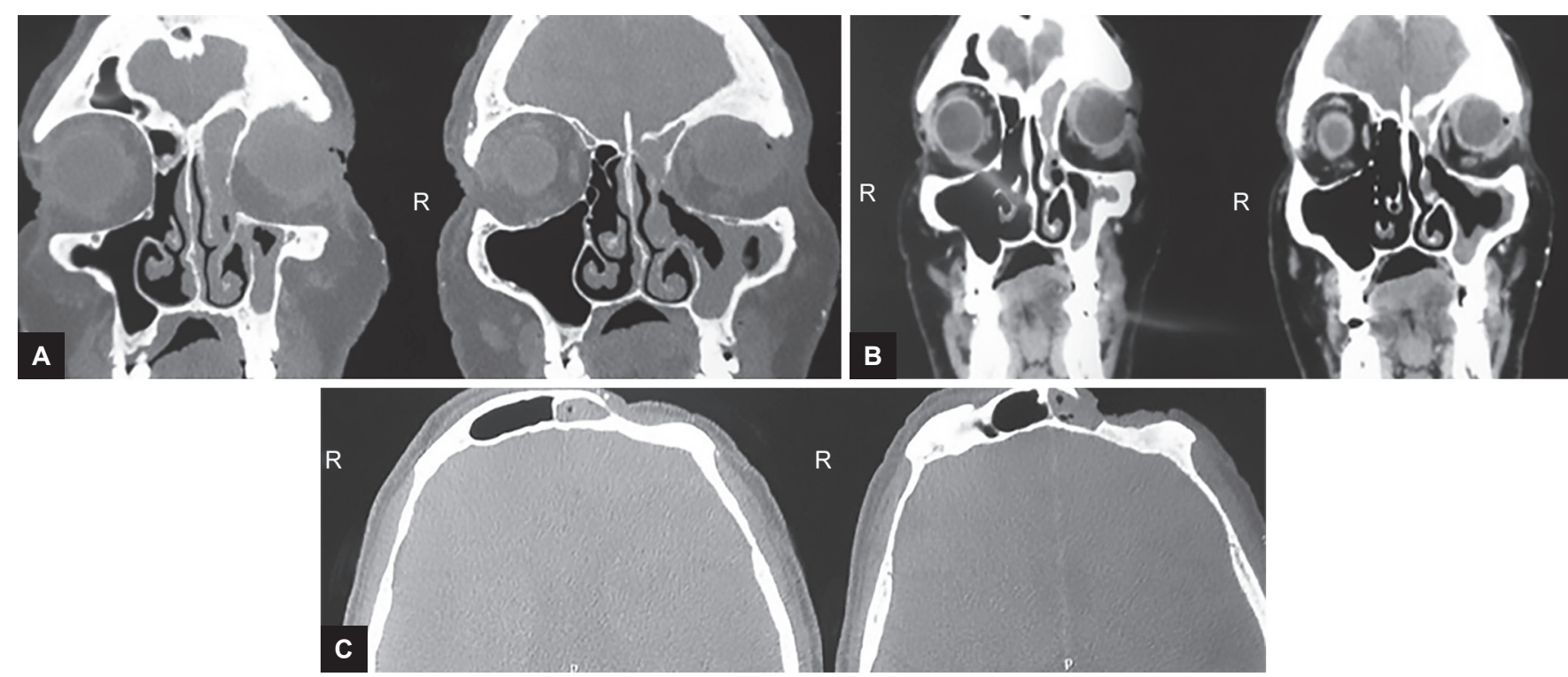

Figs $2 A$ to $C$ : Contrast-enhanced CT scans, coronal views $(A, B)$ and axial view $(C)$ showing osteolytic, peripherally enhancing soft tissue in the left frontal sinus extending to the left ethmoids with a focal defect of the left lamina papyracea, along with thickening and enhancement of the extraocular muscles of the left orbit. A defect in the supero medial aspect of left frontal sinus communicating with the overlying skin and soft tissue is also visible
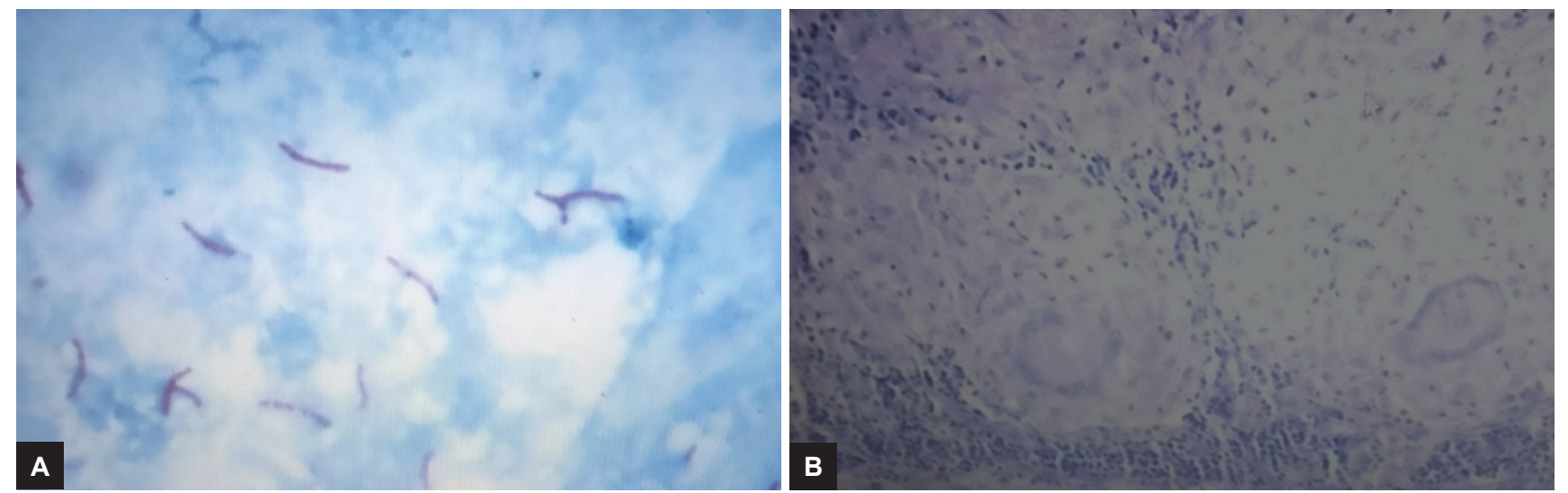

Figs 3A and B: Photomicrograph showing acid fast bacilli on Ziehl-Neelsen staining (3A), and caseating granulomas with epithelioid and Langhan's giant cells on histopathology 400× magnification (3B) 


\section{DISCUSSION}

Tuberculosis is a common public health problem in India and has varied presentations. Skeletal tuberculosis accounts for 1 to $3 \%$ of total cases of tuberculosis, out of which 0.2 to $1.3 \%$ involve the calvarial bones, ${ }^{1-3}$ thus making it extremely rare, probably owing to the peculiar blood supply. ${ }^{4,5}$ Calvarial tuberculosis usually occurs due to hematogenous spread from a primary focus elsewhere. A rare cause can be direct inoculation of the bone during surgery or penetrating injury. ${ }^{1}$ Focus in the skull starts in the diploe and then may extend to either tables; outer table involvement leads to a sinus formation, whereas inner table involvement results in extradural granulation tissue or collection. The dura usually forms a protective barrier, preventing further spread. ${ }^{1}$

The final presentation depends on the virulence of the organism and patient immunity, and may often be difficult to diagnose and treat. Radiology gives a good idea for presumptive diagnosis, but the confirmation is to be made only on microbiological and/or histopathological studies.

A computed tomography of the calvarial bones will usually reveal osteolytic lesions, sequestrum formation, sinuses, extradural collections, or granulomas, and rarely sclerotic lesions. Spread or extension to the adjacent structures like the orbit, rest of the paranasal sinuses, or calvarial bones may be noted. ${ }^{6}$ Discharge from the sinuses would reveal the presence of acid-fast bacilli on Ziehl-Neelsen staining. Isolation of mycobacteria on culture may often be tedious and time consuming and not always warranted, but is nonetheless diagnostic, and may additionally help in deciding the definitive treatment, depending on the drug sensitivity. Scrapings from the sinus walls or surgically excised specimen can be subjected to histopathology which would show caseating granulomas, epithelioid, and Langhans giant cells, and the characteristic cellular infiltrate.

Once diagnosed, the current management guidelines include a prompt initiation of antituberculous drugs which is the main modality of treatment. Treatment can be monitored using clinical and radiological response and culture studies wherever indicated. Treatment is usually given for at least a period of 6 to 9 months; however, various regimens are in place for the type and duration of therapy and may differ as per institutional protocols and the clinical, laboratory, and radiological parameters.? Surgical intervention is rarely required, except in cases of subperiosteal abscess, sequestrectomy, or for any lesion causing neurovascular compression. Surgery could be attempted in rare cases where medical therapy fails to control the disease.

\section{CONCLUSION}

Tuberculosis of the skull, though rare, must be suspected in atypical presentations as a differential diagnosis, especially in an endemic country. Clinical, radiological, microbiological, and histopathological correlation is the cornerstone for diagnosis and adequate treatment.

\section{REFERENCES}

1. Vijay S, Jitendra S, Ashok G, Virendra S. Post traumatic tubercular osteomyelitis of skull vault. J Neurosci Rural Pract 2013 Aug;4(Suppl 1):S138-S141.

2. Mehdi B, Berriche A, Ammari L, Adbelmalek R, Kilani B, Tiouiri H. Tuberculous frontal bone osteomyelitis. Ann Clin Case Rep 2017 Feb;2:Article 1274

3. Pandey AS, Surana A. Tuberculosis osteomyelitis of the bone flap following craniotomy. Indian J Tuberc 2011 Jul;58(3):129-131.

4. Alam SK, Zahid M, Brijesh S, Azra SH. Tuberculosis of frontal bone: a case report. Indian J Tuberc 2001;48:95.

5. Chambers AA, Lukin RR, Tomsick TA. Cranial and intracranial tuberculsis. Semin Koentgenol 1979 Oct;14(4):319.

6. Raut AA, Nagar AM, Muzumdar D, Chawla AJ, Narlawar RS, Fattepurkar S, Bhatgadde VL. Imaging features of calvarial tuberculosis: a study of 42 cases. AJNR Am J Neuroradiol 2004 Mar;25(3):409-414.

7. INDEX, TB Guidelines. Guidelines on extra-pulmonary tuberculosis for India. Central TB Division. Ministry of Health and Family Welfare, Government of India. 